\title{
Infinite log-concavity: developments and conjectures
}

\author{
Peter R. W. McNamara ${ }^{1}$ and Bruce E. Sagan ${ }^{2}$ \\ ${ }^{1}$ Department of Mathematics, Bucknell University, Lewisburg, PA 17837, USA \\ peter.mcnamara@bucknell. edu \\ ${ }^{2}$ Department of Mathematics, Michigan State University, East Lansing, MI 48824-1027, USA \\ sagan@math.msu.edu
}

\begin{abstract}
Given a sequence $\left(a_{k}\right)=a_{0}, a_{1}, a_{2}, \ldots$ of real numbers, define a new sequence $\mathcal{L}\left(a_{k}\right)=\left(b_{k}\right)$ where $b_{k}=a_{k}^{2}-a_{k-1} a_{k+1}$. So $\left(a_{k}\right)$ is log-concave if and only if $\left(b_{k}\right)$ is a nonnegative sequence. Call $\left(a_{k}\right)$ infinitely log-concave if $\mathcal{L}^{i}\left(a_{k}\right)$ is nonnegative for all $i \geq 1$. Boros and Moll conjectured that the rows of Pascal's triangle are infinitely log-concave. Using a computer and a stronger version of log-concavity, we prove their conjecture for the $n$th row for all $n \leq 1450$. We can also use our methods to give a simple proof of a recent result of Uminsky and Yeats about regions of infinite log-concavity. We investigate related questions about the columns of Pascal's triangle, $q$-analogues, symmetric functions, real-rooted polynomials, and Toeplitz matrices. In addition, we offer several conjectures.
\end{abstract}

Résumé. Étant donné une suite $\left(a_{k}\right)=a_{0}, a_{1}, a_{2}, \ldots$ de nombres réels, on définit une nouvelle suite $\mathcal{L}\left(a_{k}\right)=\left(b_{k}\right)$ où $b_{k}=a_{k}^{2}-a_{k-1} a_{k+1}$. Alors $\left(a_{k}\right)$ est log-concave si et seulement si $\left(b_{k}\right)$ est une suite non négative. On dit que $\left(a_{k}\right)$ est infiniment log-concave si $\mathcal{L}^{i}\left(a_{k}\right)$ est non négative pour tout $i \geq 1$. Boros et Moll ont conjecturé que les lignes du triangle de Pascal sont infiniment log-concave. Utilisant un ordinateur et une version plus forte de log-concavité, on vérifie leur conjecture pour la nième ligne, pour tout $n \leq 1450$. On peut aussi utiliser nos méthodes pour donner une preuve simple d'un résultat récent de Uminsky et Yeats à propos des régions de log-concavité infini. Reliées à ces idées, on examine des questions à propos des colonnes du triangle de Pascal, des $q$-analogues, des fonctions symétriques, des polynômes avec racines réelles, et des matrices de Toeplitz. De plus, on offre plusieurs conjectures.

Keywords: binomial coefficients, computer proof, Gaussian polynomial, infinite log-concavity, symmetric functions, real roots

\section{Introduction}

Let

$$
\left(a_{k}\right)=\left(a_{k}\right)_{k \geq 0}=a_{0}, a_{1}, a_{2}, \ldots
$$

be a sequence of real numbers. It will be convenient to extend the sequence to negative indices by letting $a_{k}=0$ for $k<0$. Also, if $\left(a_{k}\right)=a_{0}, a_{1}, \ldots, a_{n}$ is a finite sequence then we let $a_{k}=0$ for $k>n$.

Define the $\mathcal{L}$-operator on sequences to be $\mathcal{L}\left(a_{k}\right)=\left(b_{k}\right)$ where $b_{k}=a_{k}^{2}-a_{k-1} a_{k+1}$. Call a sequence $i$-fold log-concave if $\mathcal{L}^{i}\left(a_{k}\right)$ is a nonnegative sequence. So log-concavity in the ordinary sense is 1-fold 
log-concavity. Log-concave sequences arise in many areas of algebra, combinatorics, and geometry. See the survey articles of Stanley (20) and Brenti (7) for more information.

Boros and Moll (4, page 157) defined $\left(a_{k}\right)$ to be infinitely log-concave if it is $i$-fold log-concave for all $i \geq 1$. They introduced this definition in conjunction with the study of a specialization of the Jacobi polynomials whose coefficient sequence they conjectured to be infinitely log-concave. Kauers and Paule (13) used a computer algebra package to prove this conjecture for ordinary log-concavity. Since the coefficients of these polynomials can be expressed in terms of binomial coefficients, Boros and Moll also made the statement:

"Prove that the binomial coefficients are $\infty$-logconcave."

We will take this to be a conjecture that the rows of Pascal's triangle are infinitely log-concave, although we will later discuss the columns and other lines. When given a function of more than one variable, we will subscript the $\mathcal{L}$-operator by the parameter which is varying to form the sequence. So $\mathcal{L}_{k}\left(\begin{array}{l}n \\ k\end{array}\right)$ would refer to the operator acting on the sequence $\left(\begin{array}{l}n \\ k\end{array}\right)_{k>0}$. Note that we drop the sequence parentheses for sequences of binomial coefficients to improve readability. We now restate the Boros-Moll conjecture formally.

Conjecture 1.1 The sequence $\left(\begin{array}{l}n \\ k\end{array}\right)_{k \geq 0}$ is infinitely log-concave for all $n \geq 0$.

In the next section, we use a strengthened version of log-concavity and computer calculations to verify Conjecture 1.1 for all $n \leq 1450$. Uminsky and Yeats (25) set up a correspondence between certain symmetric sequences and points in $\mathbb{R}^{m}$. They then described an infinite region $\mathcal{R} \subset \mathbb{R}^{m}$ bounded by hypersurfaces and such that each sequence corresponding to a point of $\mathcal{R}$ is infinitely log-concave. In Section 3, we indicate how our methods can be used to give a simple derivation of one of their main theorems. We investigate infinite log-concavity of the columns and other lines of Pascal's triangle in Section 4 Section 5 is devoted to two $q$-analogues of the binomial coefficients. For the Gaussian polynomials, we show that certain analogues of some infinite log-concavity conjectures are false while others appear to be true. In contrast, our second $q$-analogue seems to retain all the log-concavity properties of the binomial coefficients. In Section 6, after showing why the sequence $\left(h_{k}\right)_{k \geq 0}$ of complete homogeneous symmetric is an appropriate analogue of sequences of binomial coefficients, we explore its log-concavity properties. We end with a section of related results and questions about real-rooted polynomials and Toeplitz matrices.

While one purpose of this article is to present our results, we have written it with two more targets in mind. The first is to convince our audience that infinite log-concavity is a fundamental concept. We hope that its definition as a natural extension of traditional log-concavity helps to make this case. Our second aspiration is to attract others to work on the subject; to that end, we have presented several open problems. These conjectures each represent fundamental questions in the area, so even solutions of special cases may be interesting.

\section{Rows of Pascal's triangle}

One of the difficulties with proving the Boros-Moll conjecture is that log-concavity is not preserved by the $\mathcal{L}$-operator. For example, the sequence $4,5,4$ is log-concave but $\mathcal{L}(4,5,4)=16,9,16$ is not. So we will seek a condition stronger than log-concavity which is preserved by $\mathcal{L}$. Given $r \in \mathbb{R}$, we say that a 
sequence $\left(a_{k}\right)$ is $r$-factor log-concave if

$$
a_{k}^{2} \geq r a_{k-1} a_{k+1}
$$

for all $k$. Clearly this implies log-concavity if $r \geq 1$.

We seek an $r>1$ such that $\left(a_{k}\right)$ being $r$-factor log-concave implies that $\left(b_{k}\right)=\mathcal{L}\left(a_{k}\right)$ is as well. Assume the original sequence is nonnegative. Then expanding $r b_{k-1} b_{k+1} \leq b_{k}^{2}$ in terms of the $a_{k}$ and rearranging the summands, we see that this is equivalent to proving

$$
(r-1) a_{k-1}^{2} a_{k+1}^{2}+2 a_{k-1} a_{k}^{2} a_{k+1} \leq a_{k}^{4}+r a_{k-2} a_{k}\left(a_{k+1}^{2}-a_{k} a_{k+2}\right)+r a_{k-1}^{2} a_{k} a_{k+2} .
$$

By our assumptions, the two expressions with factors of $r$ on the right are nonnegative, so it suffices to prove the inequality obtained when these are dropped. Applying 2.1] to the left-hand side gives

$$
(r-1) a_{k-1}^{2} a_{k+1}^{2}+2 a_{k-1} a_{k}^{2} a_{k+1} \leq \frac{r-1}{r^{2}} a_{k}^{4}+\frac{2}{r} a_{k}^{4}
$$

So we will be done if

$$
\frac{r-1}{r^{2}}+\frac{2}{r}=1
$$

Finding the root $r_{0}>1$ of the corresponding quadratic equation finishes the proof of the first assertion of the following lemma, while the second assertion follows easily from the first.

Lemma 2.1 Let $\left(a_{k}\right)$ be a nonnegative sequence and let $r_{0}=(3+\sqrt{5}) / 2$. Then $\left(a_{k}\right)$ being $r_{0}$-factor log-concave implies that $\mathcal{L}\left(a_{k}\right)$ is too. So in this case $\left(a_{k}\right)$ is infinitely log-concave.

Now to prove that any row of Pascal's triangle is infinitely log-concave, one merely lets a computer find $\mathcal{L}_{k}^{i}\left(\begin{array}{l}n \\ k\end{array}\right)$ for $i$ up to some bound $I$. If these sequences are all nonnegative and $\mathcal{L}_{k}^{I}\left(\begin{array}{l}n \\ k\end{array}\right)$ is $r_{0}$-factor logconcave, then the previous lemma shows that this row is infinitely log-concave. Using this technique, we have obtained the following theorem.

Theorem 2.2 The sequence $\left(\begin{array}{l}n \\ k\end{array}\right)_{k \geq 0}$ is infinitely log-concave for all $n \leq 1450$.

We note that the necessary value of $I$ increases slowly with increasing $n$. As an example, when $n=$ 100 , our technique works with $I=5$, while for $n=1000$, we need $I=8$.

Of course, the method developed in this section can be applied to any sequence such that $\mathcal{L}^{i}\left(a_{k}\right)$ is $r_{0}$-factor log-concave for some $i$. In particular, it is interesting to try it on the original sequence which motivated Boros and Moll (4) to define infinite log-concavity. They were studying the polynomial

$$
P_{m}(x)=\sum_{\ell=0}^{m} d_{\ell}(m) x^{\ell}
$$

where

$$
d_{\ell}(m)=\sum_{j=\ell}^{m} 2^{j-2 m}\left(\begin{array}{c}
2 m-2 j \\
m-j
\end{array}\right)\left(\begin{array}{c}
m+j \\
m
\end{array}\right)\left(\begin{array}{l}
j \\
\ell
\end{array}\right) .
$$

Kauers [private communication] has used our technique to verify infinite log-concavity of the sequence $\left(d_{\ell}(m)\right)_{\ell \geq 0}$ for $m \leq 129$. For such values of $m, \mathcal{L}_{\ell}^{5}$ applied to the sequence is $r_{0}$-factor $\log$-concave. 


\section{A region of infinite log-concavity}

Uminsky and Yeats (25) took a different approach to the Boros-Moll Conjecture as described in the Introduction. Since they were motivated by the rows of Pascal's triangle, they only considered real sequences $a_{0}, a_{1}, \ldots, a_{n}$ which are symmetric (in that $a_{k}=a_{n-k}$ for all $k$ ) and satisfy $a_{0}=a_{n}=1$. Each such sequence corresponds to a point $\left(a_{1}, \ldots, a_{m}\right) \in \mathbb{R}^{m}$ where $m=\lfloor n / 2\rfloor$.

Their region, $\mathcal{R}$, whose points all correspond to infinitely log-concave sequences, is bounded by $m$ parametrically defined hypersurfaces. The parameters are $x$ and $d_{1}, d_{2}, \ldots, d_{m}$ and it will be convenient to have the notation

$$
s_{k}=\sum_{i=1}^{k} d_{i}
$$

We will also need $r_{1}=(1+\sqrt{5}) / 2$. Note that $r_{1}^{2}=r_{0}$. The $k$ th hypersurface, $1 \leq k<m$, is defined as

$$
\begin{aligned}
\mathcal{H}_{k}=\left\{\left(x^{s_{1}}, \ldots, x^{s_{k-1}}, r_{1} x^{s_{k}}, x^{s_{k+1}+d_{k}-d_{k+1}}, \ldots, x^{s_{m}+d_{k}-d_{k+1}}\right):\right. \\
\left.x \geq 1, \quad 1=d_{1}>\cdots>d_{k}>d_{k+2}>\cdots>d_{m}>0\right\},
\end{aligned}
$$

while

$$
\mathcal{H}_{m}=\left\{\left(x^{s_{1}}, \ldots, x^{s_{m-1}}, c x^{s_{m-1}}\right): x \geq 1, \quad 1=d_{1}>\cdots>d_{m-1}>0\right\}
$$

where

$$
c= \begin{cases}r_{1} & \text { if } n=2 m, \\ 2 & \text { if } n=2 m+1 .\end{cases}
$$

Let us say that the correct side of $\mathcal{H}_{k}$ for $1 \leq k \leq m$ consists of those points in $\mathbb{R}^{m}$ that can be obtained from a point on $\mathcal{H}_{k}$ by increasing the $k$ th coordinate. Then let $\mathcal{R}$ be the region of all points in $\mathbb{R}^{m}$ having increasing coordinates and lying on the correct side of $\mathcal{H}_{k}$ for all $k$. Our ideas of the previous section can be used to give a simple proof of one of Uminsky and Yeats' main theorems.

Theorem 3.1 ((25)) Any sequence corresponding to a point of $\mathcal{R}$ is infinitely log-concave.

The proof relies on the fact that, since $r_{1}^{2}=r_{0}$, the conditions for containment in $\mathcal{R}$ are very close to the conditions for $r_{0}$-factor log-concavity.

\section{Columns and other lines of Pascal's triangle}

While we have treated Boros and Moll's statement about the infinite log-concavity of the binomial coefficients to be a statement about the rows of Pascal's triangle, their wording also suggests an examination of the columns.

Conjecture 4.1 The sequence $\left(\begin{array}{l}n \\ k\end{array}\right)_{n \geq k}$ is infinitely log-concave for all fixed $k \geq 0$.

We will give two pieces of evidence for this conjecture. First, it is not difficult to show infinite logconcavity for specific small values of $k$.

Proposition 4.2 The sequence $\left(\begin{array}{l}n \\ k\end{array}\right)_{n \geq k}$ is infinitely log-concave for $0 \leq k \leq 2$.

Secondly, some careful analysis shows that $\mathcal{L}_{n}^{i}\left(\begin{array}{l}n \\ k\end{array}\right)$ is nonnegative for certain values of $i$ and all $k$.

Proposition 4.3 The sequence $\mathcal{L}_{n}^{i}\left(\begin{array}{l}n \\ k\end{array}\right)$ is nonnegative for all $k$ and for $0 \leq i \leq 4$. 
Kauers and Paule (13) proved that the rows of Pascal's triangle are $i$-fold log-concave for $i \leq 5$. Kauers [private communication] has used their techniques to confirm Proposition 4.3 and to also check the case $i=5$ for the columns. For the latter case, Kauers used a computer to determine

$$
\frac{\left(\mathcal{L}_{n}^{5}\left(\begin{array}{l}
n \\
k
\end{array}\right)\right)}{\left(\begin{array}{l}
n \\
k
\end{array}\right)^{2^{5}}}
$$

explicitly, which is just a rational function in $n$ and $k$. He then showed that 4.1 is nonnegative by means of cylindrical algebraic decomposition. We refer the interested reader to (13) and the references therein for more information on such techniques.

More generally, we can look at an arbitrary line in Pascal's triangle, i.e., consider the sequence

$$
\left(\begin{array}{l}
n+m u \\
k+m v
\end{array}\right)_{m \geq 0}
$$

The unimodality and (1-fold) log-concavity of such sequences has been investigated in (3; 22; 23; 24). We do not require that $u$ and $v$ be coprime, so such sequences need not contain all of the binomial coefficients in which a geometric line would intersect Pascal's triangle, e.g., a sequence such as $\left(\begin{array}{c}n \\ 0\end{array}\right),\left(\begin{array}{c}n \\ 2\end{array}\right),\left(\begin{array}{c}n \\ 4\end{array}\right), \ldots$ would be included. By letting $u<0$, one can get a finite truncation of a column. For example, if $n=5$, $k=3, u=-1$, and $v=0$ then we get the sequence

$$
\left(\begin{array}{l}
5 \\
3
\end{array}\right),\left(\begin{array}{l}
4 \\
3
\end{array}\right),\left(\begin{array}{l}
3 \\
3
\end{array}\right)
$$

which is not even 2 -fold log-concave. So we will only consider $u \geq 0$. Also

$$
\left(\begin{array}{c}
n+m u \\
k+m v
\end{array}\right)=\left(\begin{array}{c}
n+m u \\
n-k+m(u-v)
\end{array}\right)
$$

so we can also assume $v \geq 0$.

We offer the following conjecture, which includes Conjecture 1.1 as a special case.

Conjecture 4.4 Suppose that $u$ and $v$ are distinct nonnegative integers. Then $\left(\begin{array}{c}n+m u \\ m v\end{array}\right)_{m \geq 0}$ is infinitely log-concave for all $n \geq 0$ if and only if $u<v$ or $v=0$.

We first give a quick proof of the "only if" direction. Supposing that $u>v \geq 1$, we consider the sequence

$$
\left(\begin{array}{l}
0 \\
0
\end{array}\right),\left(\begin{array}{l}
u \\
v
\end{array}\right),\left(\begin{array}{l}
2 u \\
2 v
\end{array}\right), \ldots
$$

obtained when $n=0$. We claim that this sequence is not even log-concave and that log-concavity fails at the second term. Indeed, the fact that $\left(\begin{array}{l}u \\ v\end{array}\right)^{2}<\left(\begin{array}{l}2 u \\ 2 v\end{array}\right)$ follows immediately from the identity

$$
\left(\begin{array}{l}
u \\
0
\end{array}\right)\left(\begin{array}{c}
u \\
2 v
\end{array}\right)+\left(\begin{array}{l}
u \\
1
\end{array}\right)\left(\begin{array}{c}
u \\
2 v-1
\end{array}\right)+\cdots+\left(\begin{array}{l}
u \\
v
\end{array}\right)\left(\begin{array}{l}
u \\
v
\end{array}\right)+\cdots+\left(\begin{array}{c}
u \\
2 v
\end{array}\right)\left(\begin{array}{l}
u \\
0
\end{array}\right)=\left(\begin{array}{l}
2 u \\
2 v
\end{array}\right)
$$

which is a special case of Vandermonde's Convolution. 
The proof just given shows that subsequences of the columns of Pascal's triangle are the only infinite sequences of the form $\left(\begin{array}{c}n+m u \\ m v\end{array}\right)_{m \geq 0}$ that can possibly be infinitely log-concave. We also note that the previous conjecture says nothing about what happens on the diagonal $u=v$. Of course, the case $u=v=$ 1 is Conjecture 4.1. For other diagonal values, the evidence is conflicting. One can show by computer that $\left(\begin{array}{c}n+m u \\ m u\end{array}\right)_{m \geq 0}$ is not 4 -fold log-concave for $n=2$ and any $2 \leq u \leq 500$. However, this is the only known value of $n$ for which $\left(\begin{array}{c}n+m u \\ m u\end{array}\right)_{m \geq 0}$ is not an infinitely log-concave sequence for some $u \geq 1$.

We conclude this section by offering considerable computational evidence in favor of the "if" direction of Conjecture 4.4. Theorem 2.2 provides such evidence when $u=0$ and $v=1$. Since all other sequences with $u<v$ have a finite number of nonzero entries, we can use the $r_{0}$-factor log-concavity technique for these sequences as well. For all $n \leq 500,2 \leq v \leq 20$ and $0 \leq u<v$, we have checked that $\left(\begin{array}{c}n+m u \\ m v\end{array}\right)_{m \geq 0}$ is infinitely log-concave.

\section{$5 q$-analogues}

This section will be devoted to discussing two $q$-analogues of binomial coefficients. For the Gaussian polynomials, we will see that the corresponding generalization of Conjecture 1.1 is false, and we show one exact reason why it fails. In contrast, the corresponding generalization of Conjecture 4.1 appears to be true. This shows how delicate these conjectures are and may in part explain why they seem to be difficult to prove. After introducing our second $q$-analogue, we conjecture that the corresponding generalizations of Conjectures 1.1, 4.1 and 4.4 are all true. This second $q$-analogue arises in the study of quantum groups; see, for example, the books of Jantzen (12) and Majid (17).

Let $q$ be a variable and consider a polynomial $f(q) \in \mathbb{R}[q]$. Call $f(q) q$-nonnegative if all the coefficients of $f(q)$ are nonnegative. Apply the $\mathcal{L}$-operator to sequences of polynomials $\left(f_{k}(q)\right)$ in the obvious way. Call such a sequence $q$-log-concave if $\mathcal{L}\left(f_{k}(q)\right)$ is a sequence of $q$-nonnegative polynomials, with $i$-fold $q$-log-concavity and infinite $q$-log-concavity defined similarly.

We will be particularly interested in the Gaussian polynomials. The standard q-analogue of the nonnegative integer $n$ is

$$
[n]=[n]_{q}=\frac{1-q^{n}}{1-q}=1+q+q^{2}+\cdots+q^{n-1} .
$$

Then, for $0 \leq k \leq n$, the Gaussian polynomials or $q$-binomial coefficients are defined as

$$
\left[\begin{array}{l}
n \\
k
\end{array}\right]=\left[\begin{array}{l}
n \\
k
\end{array}\right]_{q}=\frac{[n]_{q} !}{[k]_{q} ![n-k]_{q} !}
$$

where $[n]_{q} !=[1]_{q}[2]_{q} \cdots[n]_{q}$. For more information, including proofs of the assertions made in the next paragraph, see the book of Andrews (2).

Clearly substituting $q=1$ gives $\left[\begin{array}{l}n \\ k\end{array}\right]_{1}=\left(\begin{array}{l}n \\ k\end{array}\right)$. Also, it is well known that the Gaussian polynomials are indeed $q$-nonnegative polynomials. In fact, they have various combinatorial interpretations, one of which we use. An (integer) partition of $n$ is a weakly decreasing positive integer sequence $\lambda=\left(\lambda_{1}, \lambda_{2}, \ldots, \lambda_{\ell}\right)$ such that $|\lambda| \stackrel{\text { def }}{=} \sum_{i} \lambda_{i}=n$. The $\lambda_{i}$ are called parts. We say that $\lambda$ fits inside an $s \times t$ box if $\lambda_{1} \leq t$ and $\ell \leq s$. Denote the set of all such partitions by $P(s, t)$. It is well known, and easy to prove by induction on $n$, that

$$
\left[\begin{array}{l}
n \\
k
\end{array}\right]=\sum_{\lambda \in P(n-k, k)} q^{|\lambda|}
$$


Using this combinatorial interpretation, we can prove that the $q$-analogue of the rows of Pascal's triangle are not 2-fold $q$-log-concave. More specifically, we have the following result. From this point on, we use the notation $L\left(a_{k}\right)$ for the $k$ th element of the sequence $\mathcal{L}\left(a_{k}\right)$, and similarly for $L_{k}$ and $L_{n}$.

Proposition 5.1 For $n \geq 2$ and $k=\lfloor n / 2\rfloor$ we have

$$
L_{k}^{2}\left(\left[\begin{array}{l}
n \\
k
\end{array}\right]\right)=-q^{n-2}+\text { higher order terms. }
$$

Consequently, $\left(\left[\begin{array}{l}n \\ k\end{array}\right]\right)_{k \geq 0}$ is not 2-fold $q$-log-concave.

Given this, it may seem surprising that the following conjecture, which is a $q$-analogue of Conjecture 4.1, does seem to hold.

Conjecture 5.2 The sequence $\left(\left[\begin{array}{l}n \\ k\end{array}\right]\right)_{n \geq k}$ is infinitely $q$-log-concave for all fixed $k \geq 0$.

As evidence, we give a $q$-analogue of Proposition 4.2 and an appropriate adaption of Proposition 4.3 The case $i=2$ of Proposition 5.3 (b) corresponds to the $q$-log-concavity of the $q$-Narayana numbers and is a result of $(\underline{8})$.

\section{Proposition 5.3}

(a) The sequence $\left(\left[\begin{array}{l}n \\ k\end{array}\right]\right)_{n \geq k}$ is infinitely $q$-log-concave for $0 \leq k \leq 2$.

(b) The sequence $\mathcal{L}_{n}^{i}\left(\left[\begin{array}{l}n \\ k\end{array}\right]\right)$ is $q$-nonnegative for all $k$ and for $0 \leq i \leq 2$.

We conclude our discussion of the Gaussian polynomials by considering the sequence

$$
\left(\left[\begin{array}{c}
n+m u \\
m v
\end{array}\right]\right)_{m \geq 0}
$$

for nonnegative integers $u$ and $v$, as we did in Section 4 for the binomial coefficients. When $u>v$ the sequence has an infinite number of nonzero entries. We can use 5.1 to show that the highest degree term in $\left[\begin{array}{c}n+u \\ v\end{array}\right]^{2}-\left[\begin{array}{c}n+2 u \\ 2 v\end{array}\right]$ has coefficient -1 , so the sequence 5.2 is not even $q$-log-concave. When $u<v$, it seems to be the case that the sequence is not 2 -fold $q$-log-concave, as shown for the rows in Proposition 5.1. When $u=v$, the evidence is conflicting, reflecting the behavior of the binomial coefficients. Since setting $q=1$ in $\left[\begin{array}{c}n+m u \\ m u\end{array}\right]$ yields $\left(\begin{array}{c}n+m u \\ m u\end{array}\right)$, we know that $\left(\left[\begin{array}{c}2+m u \\ m u\end{array}\right]\right)_{m \geq 0}$ is not always 4 -fold $q$-log-concave. It also transpires that the case $n=3$ is not always 5 -fold $q$-log-concave. We have not encountered other values of $n$ that fail to yield a $q$-log-concave sequence when $u=v$.

While the variety of behavior of the Gaussian polynomials is interesting, it would be desirable to have a $q$-analogue that better reflects the behavior of the binomial coefficients. A $q$-analogue that arises in the study of quantum groups serves this purpose. Let us replace the previous $q$-analogue of the nonnegative integer $n$ with the expression

$$
\langle n\rangle=\frac{q^{n}-q^{-n}}{q-q^{-1}}=q^{1-n}+q^{3-n}+q^{5-n}+\cdots+q^{n-1} .
$$

From this, we obtain a $q$-analogue of the binomial coefficients by proceeding as for the Gaussian polynomials: for $0 \leq k \leq n$, we define

$$
\left\langle\begin{array}{l}
n \\
k
\end{array}\right\rangle=\frac{\langle n\rangle !}{\langle k\rangle !\langle n-k\rangle !}
$$


where $\langle n\rangle !=\langle 1\rangle\langle 2\rangle \cdots\langle n\rangle$.

Letting $q \rightarrow 1$ in $\left\langle\begin{array}{l}n \\ k\end{array}\right\rangle$ gives $\left(\begin{array}{l}n \\ k\end{array}\right)$, and a straightforward calculation shows that

$$
\left\langle\begin{array}{l}
n \\
k
\end{array}\right\rangle=\frac{1}{q^{n k-k^{2}}}\left[\begin{array}{l}
n \\
k
\end{array}\right]_{q^{2}} .
$$

So $\left\langle\begin{array}{l}n \\ k\end{array}\right\rangle$ is , in general, a Laurent polynomial in $q$ with nonnegative coefficients. Our definitions of $q$ nonnegativity and $q$-log-concavity for polynomials in $q$ extend to Laurent polynomials in the obvious way.

We offer the following generalizations of Conjectures $1.1,4.1$ and 4.4 .

\section{Conjecture 5.4}

(a) The row sequence $\left(\left\langle\begin{array}{l}n \\ k\end{array}\right\rangle\right)_{k \geq 0}$ is infinitely $q$-log-concave for all $n \geq 0$.

(b) The column sequence $\left(\left\langle\begin{array}{l}n \\ k\end{array}\right\rangle\right)_{n \geq k}$ is infinitely $q$-log-concave for all fixed $k \geq 0$.

(c) For all integers $0 \leq u<v$, the sequence $\left(\left\langle\begin{array}{c}n+m u \\ m v\end{array}\right\rangle\right)_{m \geq 0}$ is infinitely $q$-log-concave for all $n \geq 0$.

Several remarks are in order. Suppose that for $f(g), g(q) \in \mathbb{R}\left[q, q^{-1}\right]$, we say $f(q) \leq g(q)$ if $g(q)-f(q)$ is $q$-nonnegative. Then the $r$-factor log-concavity ideas of Section 2 carry over to this setting, once we replace the term "log-concave" by " $q$-log-concave." Using these ideas, we have verified Conjecture 5.4 (a) for all $n \leq 53$. Even though (a) is a special case of (c), we state it separately since (a) is the $q$-generalization of the Boros-Moll conjecture, the primary motivation for this paper. As evidence for Conjecture 5.4(b), it is not hard to prove the appropriate analogue of Proposition 5.3 . Conjecture 5.4(c) has been verified for all $n \leq 24$ with $v \leq 10$. When $u>v$, we can use 5.3 to show that the lowest degree term in $\left\langle\begin{array}{c}n+u \\ v\end{array}\right\rangle^{2}-\left\langle\begin{array}{c}n+2 u \\ 2 v\end{array}\right\rangle$ has coefficient -1 , so the sequence is not even $q$-log-concave. When $u=v$, the quantum groups analogue has exactly the same behavior as we observed above for the Gaussian polynomials.

\section{Symmetric functions}

We now turn our attention to symmetric functions. We will demonstrate that the complete homogeneous symmetric functions $\left(h_{k}\right)_{k \geq 0}$ are a natural analogue of the rows and columns of Pascal's triangle. We show that the sequence $\left(h_{k}\right)_{k \geq 0}$ is $i$-fold log-concave in the appropriate sense for $i \leq 3$, but not 4fold log-concave. Like the results of Section 5, this result underlines the difficulties and subtleties of Conjectures 1.1 and 4.1. In particular, it shows that any proof of Conjecture 1.1 or Conjecture 4.1 would need to use techniques that do not carry over to the sequence $\left(h_{k}\right)_{k \geq 0}$. For a more detailed exposition of the background material below, we refer the reader to the texts of Macdonald (16), Sagan (19) or Stanley (21).

Let $\mathbf{x}=\left\{x_{1}, x_{2}, \ldots\right\}$ be a countably infinite set of variables. For each $n \geq 0$, the elements of the symmetric group $\mathfrak{S}_{n}$ act on formal power series $f(\mathbf{x}) \in \mathbb{R}[[\mathbf{x}]]$ by permutation of variables (where $x_{i}$ is left fixed if $i>n)$. The algebra of symmetric functions, $\Lambda(\mathbf{x})$, is the set of all series left fixed by all symmetric groups and of bounded (total) degree.

The vector space of symmetric functions homogeneous of degree $k$ has dimension equal to the number of partitions $\lambda=\left(\lambda_{1}, \ldots, \lambda_{\ell}\right)$ of $k$. We will be interested in three bases for this vector space. The 
monomial symmetric function corresponding to $\lambda, m_{\lambda}=m_{\lambda}(\mathbf{x})$, is obtained by symmetrizing the monomial $x_{1}^{\lambda_{1}} \cdots x_{\ell}^{\lambda_{\ell}}$. The $k$ th complete homogeneous symmetric function, $h_{k}$, is the sum of all monomials of degree $k$. For partitions, we then define

$$
h_{\lambda}=h_{\lambda_{1}} \cdots h_{\lambda_{\ell}} .
$$

Finally, the Schur function corresponding to $\lambda$ is

$$
s_{\lambda}=\operatorname{det}\left(h_{\lambda_{i}-i+j}\right)_{1 \leq i, j \leq \ell}
$$

Our interest will be in the sequence just mentioned $\left(h_{k}\right)_{k \geq 0}$. Let $h_{k}\left(1^{n}\right)$ denote the integer obtained by substituting $x_{1}=\cdots=x_{n}=1$ and $x_{i}=0$ for $i>n$ into $h_{k}=h_{k}(\mathbf{x})$. Then $h_{k}\left(1^{n}\right)=\left(\begin{array}{c}n+k-1 \\ k\end{array}\right)$ (the number of ways of choosing $k$ things from $n$ things with repetition) and so the above sequence becomes a column of Pascal's triangle. By the same token $h_{k}\left(1^{n-k}\right)=\left(\begin{array}{c}n-1 \\ k\end{array}\right)$ and so the sequence becomes a row.

For notational convenience, if a part $k$ is repeated $r$ times in a partition $\lambda$ then we will denote this by writing $k^{r}$ in the sequence for $\lambda$. Also, when we use $\lambda$ as a subscript we will omit the parentheses. We need a result of Kirillov (14) about the product of Schur functions, which was proved bijectively by Kleber (15) and Fulmek and Kleber (11). This result can be obtained by applying the Desnanot-Jacobi Identity—also known as Dodgson's condensation formula— to the Jacobi-Trudi matrix for $s_{k^{r+1}}$.

Theorem $6.1(11 ; 14 ; 15)$ For positive integers $k, r$ we have

$$
\left(s_{k^{r}}\right)^{2}-s_{(k-1)^{r}} s_{(k+1)^{r}}=s_{k^{r-1}} s_{k^{r+1}} .
$$

To state our result, we need one more definition. If $b_{\lambda}$ is a basis for $\Lambda(\mathbf{x})$ and $f \in \Lambda(\mathbf{x})$ then we say $f$ is $b_{\lambda}$-nonnegative if the coefficient of $b_{\lambda}$ in the expansion of $f$ is nonnegative for all partitions $\lambda$. Note that $m_{\lambda}$-nonnegativity is the natural generalization to many variables of the $q$-nonnegativity definition for $\mathbb{R}[q]$. A well-known example of an $m_{\lambda}$-nonnegative symmetric function is $s_{\mu}$, for any partition $\mu$. Thus $s_{\lambda}$-nonnegativity implies $m_{\lambda}$-nonnegativity.

Theorem 6.2 The sequence $\mathcal{L}^{i}\left(h_{k}\right)$ is $s_{\lambda}$-nonnegative for $0 \leq i \leq 3$. But the sequence $\mathcal{L}^{4}\left(h_{k}\right)$ is not $m_{\lambda}$-nonnegative.

The proof involves determining $\mathcal{L}^{i}\left(h_{k}\right)$ explicitly for $0 \leq i \leq 3$, using Theorem 6.1 and various standard facts about symmetric functions to manipulate the expressions into sums of products of Schur functions; such sums are are always $s_{\lambda}$-nonnegative. By focussing on a suitable term in the expression for $L^{4}\left(h_{k}\right)$, one obtains the second assertion of the theorem.

\section{Real roots and Toeplitz matrices}

We now consider two other settings where, in contrast to the results of the previous section, Conjecture 1.1 does seem to generalize. In fact, this may be the right level of generality to find a proof.

Let $\left(a_{k}\right)=a_{0}, a_{1}, \ldots, a_{n}$ be a finite sequence of nonnegative real numbers. It was shown by Isaac Newton that if all the roots of the polynomial $p\left[a_{k}\right] \stackrel{\text { def }}{=} a_{0}+a_{1} x+\cdots a_{n} x^{n}$ are real, then the sequence $\left(a_{k}\right)$ is log-concave. For example, since the polynomial $(1+x)^{n}$ has only real roots, the $n$th row of Pascal's triangle is log-concave. It is natural to ask if the real-rootedness property is preserved by the $\mathcal{L}$-operator. The literature includes a number of results about operations on polynomials which preserve real-rootedness; for example, see $(5 ; 6,7 ;, 18,26,27)$. 
Conjecture 7.1 Let $\left(a_{k}\right)$ be a finite sequence of nonnegative real numbers. If $p\left[a_{k}\right]$ has only real roots then the same is true of $p\left[\mathcal{L}\left(a_{k}\right)\right]$.

This conjecture is due independently to Richard Stanley [private communication]. It is also one of a number of related conjectures made by Steve Fisk (10). If true, Conjecture 7.1 would immediately imply the original Boros-Moll Conjecture. As evidence for the conjecture, we have verified it by computer for a large number of randomly chosen real-rooted polynomials. We have also checked that $p\left[\mathcal{L}_{k}^{i}\left(\begin{array}{l}n \\ k\end{array}\right)\right]$ has only real roots for all $i \leq 10$ and $n \leq 40$.

Along with the rows of Pascal's triangle, it appears that applying $\mathcal{L}$ to the other finite lines we were considering in Section 4 also yields sequences with real-rooted generating functions. So we make the following conjecture which implies the "if" direction of Conjecture 4.4

Conjecture 7.2 For $0 \leq u<v$, the polynomial $p\left[\mathcal{L}_{m}^{i}\left(\left(\begin{array}{c}n+m u \\ m v\end{array}\right)\right)\right]$ has only real roots for all $i \geq 0$.

We have verified this assertion for all $n \leq 24$ with $i \leq 10$ and $v \leq 10$. In fact, it follows from a theorem of Yu (28) that the conjecture holds for $i=0$ and all $0 \leq u<v$. So it will suffice to prove Conjecture 7.1 to obtain this result for all $i$.

We can obtain a matrix-theoretic perspective on problems of real-rootedness via the following renowned result of Aissen, Schoenberg and Whitney (1). A matrix $A$ is said to be totally nonnegative if every minor of $A$ is nonnegative. We can associate with any sequence $\left(a_{k}\right)$ a corresponding (infinite) Toeplitz matrix $A=\left(a_{j-i}\right)_{i, j \geq 0}$. In comparing the next theorem to Newton's result, note that for a real-rooted polynomial $p\left[a_{k}\right]$ the roots being nonpositive is equivalent to the sequence $\left(a_{k}\right)$ being nonnegative.

Theorem $7.3((1))$ Let $\left(a_{k}\right)$ be a finite sequence of real numbers. Then every root of $p\left[a_{k}\right]$ is a nonpositive real number if and only if the Toeplitz matrix $\left(a_{j-i}\right)_{i, j \geq 0}$ is totally nonnegative.

To make a connection with the $\mathcal{L}$-operator, note that

$$
a_{k}^{2}-a_{k-1} a_{k+1}=\left|\begin{array}{cc}
a_{k} & a_{k+1} \\
a_{k-1} & a_{k}
\end{array}\right|
$$

which is a minor of the Toeplitz matrix $A=\left(a_{j-i}\right)_{i, j \geq 0}$. Call such a minor adjacent since its entries are adjacent in $A$. Now, for an arbitrary infinite matrix $A=\left(a_{i, j}\right)_{i, j \geq 0}$, let us define the infinite matrix $\mathcal{L}(A)$ by

$$
\mathcal{L}(A)=\left(\left|\begin{array}{cc}
a_{i, j} & a_{i, j+1} \\
a_{i+1, j} & a_{i+1, j+1}
\end{array}\right|\right)_{i, j \geq 0}
$$

Note that if $A$ is the Toeplitz matrix of $\left(a_{k}\right)$ then $\mathcal{L}(A)$ is the Toeplitz matrix of $\mathcal{L}\left(a_{k}\right)$. Using Theorem7.3. Conjecture 7.1 can now be strengthened as follows.

Conjecture 7.4 For a sequence $\left(a_{k}\right)$ of real numbers, if $A=\left(a_{j-i}\right)_{i, j \geq 0}$ is totally nonnegative then $\mathcal{L}(A)$ is also totally nonnegative.

Note that if $\left(a_{k}\right)$ is finite, then Conjecture 7.4 is equivalent to Conjecture 7.1 As regards evidence for Conjecture 7.4 consider an arbitrary $n$-by- $n$ matrix $A=\left(a_{i, j}\right)_{i, j=1}^{n}$. For finite matrices, $\mathcal{L}(A)$ is defined in the obvious way to be the $(n-1)$-by- $(n-1)$ matrix consisting of the 2-by-2 adjacent minors of $A$. In (9. 
Theorem 6.5), Fallat, Herman, Gekhtman, and Johnson show that for $n \leq 4, \mathcal{L}(A)$ is totally nonnegative whenever $A$ is. However, for $n=5$, an example from their paper can be modified to show that if

$$
A=\left(\begin{array}{ccccc}
1 & t & 0 & 0 & 0 \\
t & t^{2}+1 & 2 t & t^{2} & 0 \\
t^{2} & t^{3}+2 t & 1+4 t^{2} & 2 t^{3}+t & 0 \\
0 & t^{2} & 2 t^{3}+2 t & t^{4}+2 t^{2}+1 & t \\
0 & 0 & t^{2} & t^{3}+t & t^{2}
\end{array}\right)
$$

then $A$ is totally nonnegative for $t \geq 0$, but $\mathcal{L}(A)$ is not totally nonnegative for sufficiently large $t$ ( $t \geq \sqrt{2}$ will suffice). We conclude that the Toeplitz structure would be important to any affirmative answer to Conjecture 7.4 .

\section{Acknowledgements}

We thank Bodo Lass for suggesting that we approach Conjecture 1.1 from the point-of-view of real roots of polynomials. Section 7 also benefited from interesting discussions with Charles R. Johnson.

\section{References}

[1] Michael Aissen, I. J. Schoenberg, and A. M. Whitney. On the generating functions of totally positive sequences. I. J. Analyse Math., 2:93-103, 1952.

[2] George E. Andrews. The theory of partitions. Cambridge University Press, Cambridge, 1998. Reprint of the 1976 original.

[3] Hacène Belbachir, Farid Bencherif, and László Szalay. Unimodality of certain sequences connected with binomial coefficients. J. Integer Seq., 10(2):Article 07.2.3, 9 pp. (electronic), 2007.

[4] George Boros and Victor Moll. Irresistible integrals. Cambridge University Press, Cambridge, 2004.

[5] Petter Brändén. On linear transformations preserving the Pólya frequency property. Trans. Amer. Math. Soc., 358(8):3697-3716, 2006.

[6] Francesco Brenti. Unimodal, log-concave and Pólya frequency sequences in combinatorics. Mem. Amer. Math. Soc., 81(413):viii+106, 1989.

[7] Francesco Brenti. Log-concave and unimodal sequences in algebra, combinatorics, and geometry: an update. In Jerusalem combinatorics '93, volume 178 of Contemp. Math., pages 71-89. Amer. Math. Soc., Providence, RI, 1994.

[8] William Y. C. Chen, Larry X. W. Wang, and Arthur L. B. Yang. Schur positivity and the $q$-logconvexity of the Narayana polynomials. Preprint. arXiv:0806.1561.

[9] Shaun M. Fallat, Allen Herman, Michael I. Gekhtman, and Charles R. Johnson. Compressions of totally positive matrices. SIAM J. Matrix Anal. Appl., 28(1):68-80, 2006.

[10] Steve T. Fisk. Questions about determinants of polynomials. Preprint. arXiv:0808.1850. 
[11] Markus Fulmek and Michael Kleber. Bijective proofs for Schur function identities which imply Dodgson's condensation formula and Plücker relations. Electron. J. Combin., 8(1):Research Paper 16, 22 pp. (electronic), 2001.

[12] Jens Carsten Jantzen. Lectures on quantum groups. American Mathematical Society, Providence, RI, 1996.

[13] Manuel Kauers and Peter Paule. A computer proof of Moll's log-concavity conjecture. Proc. Amer. Math. Soc., 135(12):3847-3856, 2007.

[14] A. N. Kirillov. Completeness of states of the generalized Heisenberg magnet. Zap. Nauchn. Sem. Leningrad. Otdel. Mat. Inst. Steklov. (LOMI), 134:169-189, 1984.

[15] Michael Kleber. Plücker relations on Schur functions. J. Algebraic Combin., 13(2):199-211, 2001.

[16] I. G. Macdonald. Symmetric functions and Hall polynomials. The Clarendon Press Oxford University Press, New York, second edition, 1995.

[17] Shahn Majid. A quantum groups primer. Cambridge University Press, Cambridge, 2002.

[18] Jim Pitman. Probabilistic bounds on the coefficients of polynomials with only real zeros. J. Combin. Theory Ser. A, 77(2):279-303, 1997.

[19] Bruce E. Sagan. The symmetric group: Representations, combinatorial algorithms, and symmetric functions. Springer-Verlag, New York, second edition, 2001.

[20] Richard P. Stanley. Log-concave and unimodal sequences in algebra, combinatorics, and geometry. In Graph theory and its applications: East and West (Jinan, 1986). New York Acad. Sci., New York, 1989.

[21] Richard P. Stanley. Enumerative Combinatorics. Vol. 2. Cambridge University Press, Cambridge, 1999.

[22] Xun-Tuan Su and Yi Wang. On unimodality problems in Pascal's triangle. Electron. J. Combin., 15(1):Research Paper 113, 12 pp. (electronic), 2008.

[23] S. M. Tanny and M. Zuker. On a unimodal sequence of binomial coefficients. Discrete Math., 9:79-89, 1974.

[24] Stephen M. Tanny and Michael Zuker. On a unimodal sequence of binomial coefficients. II. $J$. Combinatorics Information Syst. Sci., 1(3-4):81-91, 1976.

[25] David Uminsky and Karen Yeats. Unbounded regions of infinitely logconcave sequences. Electron. J. Combin., 14(1):Research Paper 72, 13 pp. (electronic), 2007.

[26] David G. Wagner. Total positivity of Hadamard products. J. Math. Anal. Appl., 163(2):459-483, 1992.

[27] Yi Wang and Yeong-Nan Yeh. Polynomials with real zeros and Pólya frequency sequences. $J$. Combin. Theory Ser. A, 109(1):63-74, 2005.

[28] Yaming Yu. Confirming two conjectures of Su and Wang. Preprint. arXiv:0901.0385. 